\title{
Spin Asymmetries of the Nucleon Experiment
}

\author{
Narbe Kalantarians * $^{* \dagger}$ \\ University of Virginia Charlottesville, VA 22901 \\ E-mail: narbedjlab.org
}

The Spin Asymmetries of the Nucleon Experiment (SANE) is a measurement of the double spin asymmetries $A_{1}\left(x, Q^{2}\right), A_{2}\left(x, Q^{2}\right)$ and the spin structure functions $g_{1}\left(x, Q^{2}\right), g_{2}\left(x, Q^{2}\right)$ over a broad kinematic range of Bjorken scaling variable $x, 0.3<x<0.8$, for range of four-momentum transfer $2.5<Q^{2}<6.5 \mathrm{GeV}^{2}$. The moments of $g_{1}\left(x, Q^{2}\right)$ and $g_{2}\left(x, Q^{2}\right)$ will provide access to the higher twists related to quark-gluon interactions and calculable in confinement models of QCD. The experiment measured inclusive double spin asymmetries using the TJNAF polarized electron beam with energies of 4.7 and $5.9 \mathrm{GeV}$, which was scattered off the UVa polarized solid $\mathrm{NH}_{3}$ target in both parallel and transverse configurations. The experiment took place from January to March of 2009. We will discuss the physics motivation for SANE and current status of the analysis.

XVIII International Workshop on Deep-Inelastic Scattering and Related Subjects, DIS 2010 April 19-23, 2010

Firenze, Italy

\footnotetext{
*Speaker.

†. A. Rondon, M. K. Jones, D. B. Day.
} 


\section{Motivation}

The Spin Asymmetries of the Nucleon Experiment (SANE)took data from January to March of 2009 in experimental Hall $\mathrm{C}$ to measure spin structure of the proton in the important region of intermediate to high Bjorken $x, 0.3<x<0.8$, for range of four-momentum transfer $2.5<$ $Q^{2}<6.5 \mathrm{GeV}^{2}$. where nucleon spin structure is largely unknown. The goal of SANE is to obtain a maximum amount of information about spin structure of the proton from an inclusive double polarization electron scattering measurement. Using the longitudinally polarized electron beam from the Continuous Electron Beam Accelerator Facility (CEBAF) to scatter off the UVa solid ammonia $\mathrm{NH}_{3}$ target polarized in both parallel and near perpendicular $80^{\circ}$ configurations, SANE can measure both the parallel and perpendicular asymmetries $A_{\|}$and $A_{\perp}$, respectively. From these measured asymmetries it is possible to calculate the physics asymmetries $A_{1}\left(x, Q^{2}\right)$ and $A_{2}\left(x, Q^{2}\right)$, and the spin structure functions $g_{1}\left(x, Q^{2}\right)$ and $g_{2}\left(x, Q^{2}\right)$.

One of the major contributions from SANE will be $A_{\perp}$, filling a large void of data for the proton, which only has a few data points [1], [2] having limited precision in the region $x>0.3$ and $Q^{2}<6 \mathrm{GeV}^{2}$, with the exception of the RSS measurement [3]. Using moments of $g_{1}\left(x, Q^{2}\right)$ and $g_{2}\left(x, Q^{2}\right)$ it will be possible to calculate higher twists. The Nachtmann moments will be computed over the measured $x$ range at several values of constant $Q^{2}$, in combination with other data at neighboring kinematics [1], [2], [4]. The twist-3 quark-matrix element $d_{2}\left(Q^{2}\right)$ is related to the third Nachtmann moment [5]. It is relevant to quark-gluon correlations by reflecting the color electric and magnetic field response to the spin alignment of the nucleon. The measurement of $d_{2}\left(Q^{2}\right)$ from SANE can be compared with models including the Handbag model, [6], [7], [8] chiral solitons [9], [10], QCD sum-rules [11], [12], and also lattice QCD [13].

Other important sum rules, including the Burkhardt-Cottingham [14] and Efremov-TeryaevLeader sum rules can [15] be tested with SANE measurements of $g_{1}\left(x, Q^{2}\right)$ and $g_{2}\left(x, Q^{2}\right)$. The Efremov-Teryaev-Leader sum rule would be tested in combination with neutron data from Hall-A in JLab. The data from SANE can also be used to test $x$-dependence of $g_{1}\left(x, Q^{2}\right)$ and $g_{2}\left(x, Q^{2}\right)$ predicted by nucleon models as well as studying their $Q^{2}$-dependence at fixed $x$ and invariant mass of final states $W$.

SANE data will make it possible to measure $A_{2}\left(x, Q^{2}\right)$ directly. This would also be a significant contribution from SANE as it would considerably improve the world data of this asymmetry, relevant to the case for $A_{\perp}$. This would result in a model-free measurement of $A_{1}\left(x, Q^{2}\right)$. The model-independent result for $A_{1}\left(x, Q^{2}\right)$ to $x=1$ would be compared with the predictions of valence-quark models [16].

\section{Experiment}

SANE measured inclusive double spin asymmetries for parallel and near perpendicular target field configurations relative to the longitudinal beam helicity. The polarized electrons of energies 4.7 and $5.9 \mathrm{GeV}$ scattered incident on polarized protons via the solid $\mathrm{NH}_{3}$ target. The target is polarized via dynamic nuclear polarization 17.

The scattered electrons were detected in a novel large solid angle non-magnetic electron telescope named the Big Electron Telescope Array (BETA). BETA was placed at $40^{\circ}$ relative to the 


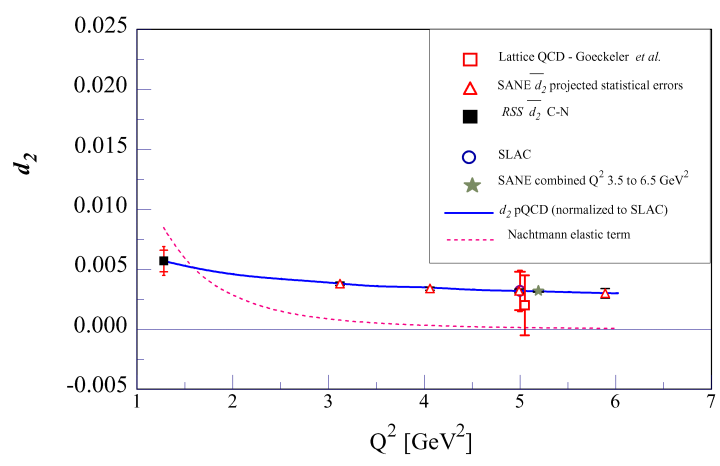

Figure 1: Expected results (statistical errors) for $\bar{d}_{2}$ (open triangles), plotted on the expected pQCD evolution of $d_{2}$ (solid curve), normalized to SLAC's C-N result (open circle) at $5 \mathrm{GeV}^{2}$. The solid square represents the RSS result. The star indicates combined SANE results for $\left\langle Q^{2}\right\rangle=5.2 \mathrm{GeV}^{2}$. The lattice QCD calculation [13] (open square) is also shown. The elastic Nachtmann and C-N contributions are indicated by the dashed and dotted curves, respectively.

\begin{tabular}{|l|c|c|c|}
\hline Configuration & $\begin{array}{c}\text { Energy } \\
{[\mathrm{GeV}]}\end{array}$ & $\begin{array}{c}<P_{\text {Beam }}> \\
{[\%]}\end{array}$ & $\begin{array}{c}<P_{\text {Target }}> \\
{[\%]}\end{array}$ \\
\hline$A_{\perp}\left(80^{\circ}\right)$ & 4.7 & 85 & 66 \\
$A_{\perp}\left(80^{\circ}\right)$ & 5.9 & 71 & 69 \\
$A_{\|}$ & 4.7 & 71 & 68 \\
$A_{\|}$ & 5.9 & 66 & 66 \\
\hline
\end{tabular}

Table 1: Summary of Collected Data for SANE. The beam polarization was determined by a Moeller polarimeter. The average target polarizations were calculated from the corrected online values.

beam direction, to cover the optimal $\left(x, Q^{2}\right)$ phase space. A summary of the data collected in the run is given in Table 2.

BETA consists of 4 subsystems/detectors. First is a lead-glass calorimeter consisting of 1792 elements. The front-face was $335 \mathrm{~cm}$ from the target. The energy is calibrated using this to detect neutral pions from events with 2 neutral clusters. A gas Cerenkov detector was used to reduce the pion background. This consists of 2 stacks of 4 mirrors reflecting light in nitrogen at atmospheric pressure, then focusing it onto 8 photomultiplier tubes (pmts) of 3" diameter. There was a lucite hodoscope composed of 28 curved horizontal bars with 2" diameter pmts at each end. This is for determination of horizontal hit position. A forward tracking hodoscope composed of fibers glued on scintillator bars was placed in front of the Cerenkov and adjacent to the target chamber. The purpose of this is to help in discerning between electron and positrons.

\section{Analysis}

Reconstruction of cluster positions and energies detected in BETA by conventional methods yielded unsatisfactory position resolution, motivating use of a novel technique known as artificial neural network (ANN) [18]. An ANN is essentially an algorithm that is trained via Monte Carlo 


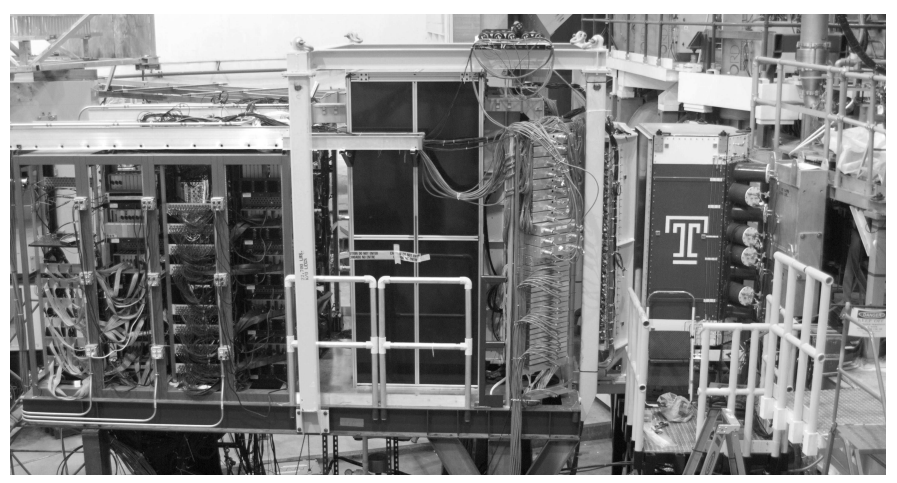

Figure 2: Side view of BETA. From left: BigCal electronics, HV box(black panels), BigCal, Lucite Hodoscope(next to Cerenkov), Cerenkov(with Temple U logo), target platforms.

(MC). The trained ANN uses cluster energy and patterns to identify the coordinate and energy of the cluster. It is seen in Figure 3 that the ANN improves on the resolution considerably.

The MC used for SANE is GEANT based [19]. In addition to training the ANN, the MC is also intended for understanding BETA's efficiencies for the purpose of obtaining the dilution factors. The packing fractions are being obtained from data that used the Hall-C High Momentum Spectrometer (HMS). This was partly due to it being very well understood for its acceptances and efficiencies. The method for obtaining the packing fractions and dilution factors is similar to what was done for RSS [20]. Some preliminary packing fractions and dilution factors have been obtained with the error calculations being in progress.

The current status of the SANE analysis is a focus on getting the preliminary $A_{\|}$. This is because the parallel data is better understood and can be used as a basis on which to check the perpendicular data. The $A_{\|}$should be finalized around the Fall of this year and the $A_{\perp}$ should take maybe a few months after that.

There are 6 doctoral students writing their dissertations on SANE: James Maxwell(UVa), Jonathan Mulholland(UVa), Whitney Armstrong(Temple), Hoyoung Kang (Seoul Natl. U), Luwani Ndukum(Miss. St. U), Anusha Liyanage(Hampton U). In addition there are also 2 master's students: Martin Jones(graduated) and John German (both NC A\&T).
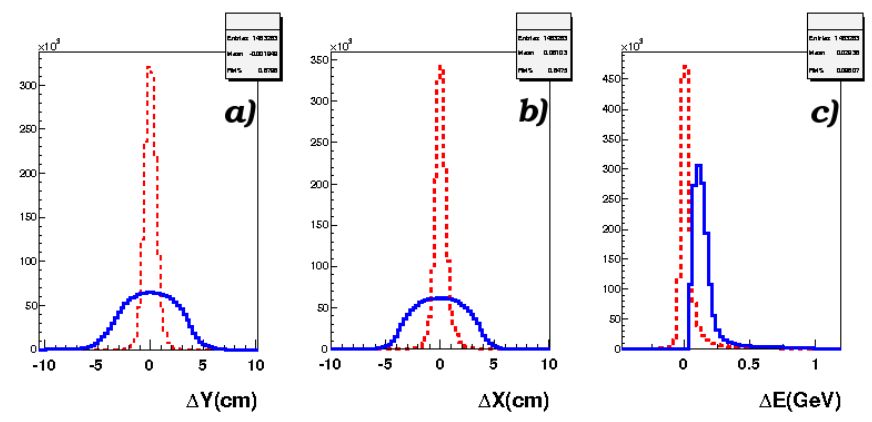

Figure 3: Difference between generated and reconstructed $Y, X$, and $E$ using conventional(solid blue curves) and $\mathrm{ANN}$ (dashed red curves) methods. 


\section{Acknowledgments}

This work was supported by the Department of Energy and by the Institute of Nuclear and Particle Physics at the University of Virginia.

\section{References}

[1] K. Abe, et al. [E143 Collaboration], Phys. Rev. D58, 112003 (1998).

[2] P. L. Anthony, et al. [E155 Collaboration], Phys. Lett. B458, 529 (1999); P. L. Anthony, et al. [E155 Collaboration], Phys. Lett. B553, 18 (2003).

[3] F. R. Wesselmann, et al. [E143 Collaboration], Phys. Rev. Lett. 98, 132003 (2007), [nucl-ex/0608003].

[4] K. V. Dharmawardane, et al. [CLAS Collaboration], Phys. Lett. B641, 11 (2007),

[5] S. Matsuda, T. Uematsu, Nucl. Phys. B168, 181 (1980).

[6] M. Stratmann, Z. Phys. C60, 763 (1993).

[7] X. Ji, P. Unrau, Phys. Lett. B333, 228 (1994).

[8] X. Song, J. S. McCarthy, Phys. Rev. D49, 3169 (1994); D50, 4718 (1994) (Erratum).

[9] H. Weigel, L. Gamberg, H. Reinhardt, Phys. Rev. D55, 6910 (1997).

[10] M. Wakamatsu, Phys. Lett. B487, 118 (2000).

[11] E. Stein, et al. Phys. Lett. B343, 369 (1995).

[12] I. Balitsky, et al. Phys. Lett. B242, 245 (1990); B318, 648 (1993) (Erratum).

[13] M. Gockeler, et al. Phys. Rev. D49, 054507 (2005).

[14] H. Burkhardt, W. N. Cottingham, Ann. Phys. 56, 453 (1970).

[15] A. V. Efremov, O. V. Teryaev, E. Leader, Phys. Rev. D55, 4307 (1997).

[16] N. Isgur, Phys. Rev. D59, 034013 (1999).

[17] D. G. Crabb, W. Meyer, Annurev. Nucl. 47, 67 (1997).

[18] V. Breton, et al., Nucl. Instrum. Meth. A362, 478 (1995).

[19] G. Warren, GEANT Simulation and Analysis Package of BETA (2003).

[20] O. A. Rondon, The Packing Fraction and Dilution Factor in RSS, RSS T. N. 2005-03 (2006). 